\title{
The TNF-NF-kB-DKK1 Axis Promoted Bone Formation in the Enthesis of Ankylosing Spondylitis
}

\author{
Sungsin Jo, Ph.D. ${ }^{1}$, Bora Nam, M.D. ${ }^{1,2}$, Young Lim Lee, M.S. ${ }^{1}$, Hyosun Park, B.S. ${ }^{1,3}$, Subin Weon, B.S. ${ }^{1,3}$, \\ Sung-Hoon Choi, M.D., Ph.D. ${ }^{4}$, Ye-Soo Park, M.D., Ph.D. ${ }^{5}$, Tae-Hwan Kim, M.D., Ph.D. ${ }^{1,2,3}$ \\ ${ }^{1}$ Hanyang University Institute for Rheumatology Research, ${ }^{2}$ Department of Rheumatology, Hanyang University Hospital for Rheumatic Diseases, \\ ${ }^{3}$ Department of Translational Medicine, Graduate School of Biomedical Science and Engineering, Hanyang University, ${ }^{4}$ Department of \\ Orthopedic Surgery, Hanyang University Hospital, Seoul, ${ }^{5}$ Department of Orthopedic Surgery, Hanyang University Guri Hospital, Hanyang \\ University College of Medicine, Guri, Korea
}

\begin{abstract}
Objective. This study aimed to determine the serum Dickkopf 1 (DKK1) levels in ankylosing spondylitis (AS) patients and decipher the mechanism of tumor necrosis factor (TNF)-mediated DKK1 regulation in human AS enthesis cells. Methods. The sera were obtained from 103 patients with AS and 30 healthy controls (HCs). The enthesis of facet joints were obtained from 4 AS patients and 5 controls. The serum levels of DKK1 were measured using ELISA and compared between AS and HCs. The impact of TNF on DKK1 expression in human primary spinal enthesis cells was evaluated using various molecular biology techniques and bone formation indicators. Results. AS patients showed higher serum DKK1 levels than HCs after adjusting for age (917.4 [615.3 1,310.0] pg/mL vs. 826.2 [670.3 927.8] pg/mL, p=0.043). TNF treatment promoted bone formation and DKK1 expression in both control enthesis cells and those of AS. This enhanced bone formation by TNF was pronounced in AS-enthesis than those of controls. Mechanically, TNF induced NF- $\kappa$ B activation upregulates the DKK1 transcript level. While, NF- $\kappa B$ inhibitor led to downregulate DKK1 expression in the enthesis. Besides, DKK1 overexpression promoted bone formation in enthesis. Conclusion. TNF induced DKK1 expression in the enthesis through NF- $\kappa$ B activation. TNF-induced DKK1 expression may play a bone formation in the radiologic progression of ankylosing spondylitis. (J Rheum Dis 2021;28:216-224)
\end{abstract}

Key Words. Ankylosing spondylitis, Tumor necrosis factor, DKK1, Enthesis, Bone formation

\section{INTRODUCTION}

Ankylosing spondylitis (AS) is a chronic inflammatory disease characterized by new bone formation that eventually leads to ankylosis of the spine and sacroiliac joints $[1,2]$. Clinically, the most striking feature of AS is enthesitis, which is characterized by inflammation of a ligament, tendon, or ligament attachment site and diffuse bone edema following soft tissue inflammation. Enthesitis is considered to have pivotal role in initiation and progression of bony ankylosis in AS [3]. However, little is known about the underlying molecular mechanism of bony ankylosis and enthesitis in AS.
Among several important pro-inflammatory cytokines, tumor necrosis factor (TNF) is thought to play a key pathophysiological role in AS. Increased expression of TNF has been reported in the serum or affected sacroiliac joint of AS patients [4,5]. Anti-TNF treatment, which is widely used in AS, not only improves clinical outcomes but also reduces radiographic progression [6]. Therefore, to improve our understating of the underlying mechanisms of bony ankylosis in AS, there is need for a thorough investigation of the enthesis response to TNF.

Dickkopf 1 (DKK1) is an inhibitory molecule of the $\mathrm{Wnt} / \beta$-catenin signaling pathway, which can stimulate new bone formation by regulating osteoblastogenesis and

Received : March 18, 2021, Revised : June 17, 2021, Accepted : June 21, 2021

Corresponding to : Tae-Hwan Kim iD http://orcid.org/0000-0002-3542-2276

Department of Rheumatology, Hanyang University Hospital for Rheumatic Diseases, 222-1 Wangsimni-ro, Seongdong-gu, Seoul 04763, Korea. E-mail : thkim@hanyang.ac.kr 
osteoclastogenesis [7]. Since new bone formation is a key pathological feature of AS, several efforts have been made to elucidate whether DKK1 is involved in pathogenesis of AS [8-11]. However, previous studies have shown controversial results regarding the serum DKK1 levels in AS patients [12,13]. In addition, the relationship between TNF and DKK1 expression in enthesis and its underlying molecular mechanism targeting bone formation of AS have not been reported so far.

In this study, we sought to determine the serum DKK-1 levels in AS patients and elucidate the underlying mechanism of TNF-mediated DKK1 expression in enthesis cells. We also reported here that DKK1 overexpression is involved in bone formation in enthesis. These results suggest that TNF-induced DKK1 expression may play an important role in pathological bone formation in AS.

\section{MATERIALS AND METHODS}

\section{Ethical approval}

This study was performed in accordance with the recommendation of institutional guidelines and the Ethics Committee of Hanyang University Hospital (IRB 201405-002) and Hanyang University Guri Hospital (IRB 2014-05-001). Written informed consent was obtained from all subjects.

\section{Patients}

We obtained sera from 103 patients with AS (mean age $45.4 \pm 5.0$ years) and 30 healthy controls (mean age $32.1 \pm 5.2$ years). All of the AS patients and healthy controls (HCs) was male. All AS patients were satisfied with modified New York criteria [14].

Facet joint entheses were obtained from four AS patients (mean age $42.1 \pm 5.7$ years, 2 males) undergoing spinal correction surgery and five controls (mean age $56 \pm 11.8$ years, 3 males) who underwent spinal surgery for non-inflammatory spinal diseases. All AS patients were HLA-B27 positive patients. Two patients (50\%) were treated with biologic agents [15].

\section{DKK1 measurement}

The human sera from the AS and HCs were collected in a separator tube and allowed to clot for an hour at $4^{\circ} \mathrm{C}$. The samples were then centrifuged for 15 minutes at approximately $3,000 \mathrm{rpm}$ at $4^{\circ} \mathrm{C}$. All sera were immediately aliquoted and stored at $-80^{\circ} \mathrm{C}$. The collected sera were tested for human DKK1 (DB100B; R\&D, Minneapolis,
MN, USA) by a commercial ELISA kit according to the manufacturer's protocols. Since the classic DKK1 standard curve ranges are from $0 \sim 2,000 \mathrm{pg} / \mathrm{mL}$, we fixed the values as $2,000 \mathrm{pg} / \mathrm{mL}$ if the DKK1 levels go over 2,000 $\mathrm{pg} / \mathrm{mL}$.

\section{Human enthesis cells and bone formation in vitro}

The isolation and characteristics of the primary enthesis were previously described [15]. Briefly, the spinal bones were repeatedly washed with pre-chilled $1 \times$ PBS twice to remove the suspension cells. The excess or adjuvant soft tissues of the bone were collected. The collected enthesis tissues were incubated in DMEM (10082147; Gibco, Waltham, MA, USA) containing collagenase type 1 (17100-017; Gibco) and antibiotics (15140-122; Gibco) with shaking. On the next day, the isolated cells were seeded and used for experiments in the second or fourth passages. Before the cell stock storage, the primary enthesis cells were checked for mycoplasma using PCR methods (6601; Takara, Kyoto, Japan). To induce bone formation, the cells were seeded in DMEM medium and then stimulated with the conditional medium including ascorbic acid, $\beta$-glycerolphosphate, and dexamethasone. The conditional medium was exchanged every three days. The experimental design and measurement methods for changes in bone formation are shown in Figure 2A.

\section{Reagents}

Recombinant human TNF (300-01A; Peprotech, Cranbury, NJ, USA) and BAY 11-7082 (B5556; Sigma, St. Louis, MO, USA) were used.

\section{Luciferase assay}

The 293T cells were co-transfected with the luciferase gene tagged human promoter and firefly plasmid using Lipofectamine 3000 (L3000008; Thermo, Waltham, MA, USA). The cells were then incubated for 48 hours and analyzed by the firefly luciferase activity (E1500; Promega, Madison, WI, USA) according to the manufacturer's protocol. Luciferase activity was measured using a Luminometer (Berthold; Bad Wildbad, Baden-Württemberg, Germany). Two types (1 kb and $0.35 \mathrm{~kb}$ ) of Human DKK1 promoters, three types (WT, S536A, S5336E) of human NF- $\kappa$ B promoters, and firefly were generously obtained from Prof. Jeong-Yeon Lee (Department of Pathology, College of Medicine, Hanyang University, Seoul, Korea) $[16,17]$. 


\section{Quantitative reverse transcriptase-PCR}

The complementary DNA were generated using reverse transcriptase (K1622; Thermo). Quantitative PCR (qPCR) was performed on a CFX96 Real-time thermocycler from Bio-Rad using iQ SYBR Green Supermix (170-8882AP; Bio-Rad, Hercules, CA, USA) according to the manufacturer's protocol. For all samples, the target gene expression was normalized to GAPDH. The oligonucleotide primer sequences are given in Table 1.

\section{Immunoblotting}

For the immunoblot analysis, the cell pellets were lysed in RIPA buffer (50 mM Tris-HCl [pH 8.0], $150 \mathrm{mM} \mathrm{NaCl}$, $0.1 \%$ SDS, $0.6 \%$ Na-deoxycholate, $1 \%$ Triton X-100) that was supplemented with protease (535140; Calbiochem, St. Louis, MO, USA) and phosphatase (5870; Cell signaling, Denvers, MA, USA) inhibitor cocktails. The lysed samples were incubated on ice for 30 minutes followed by centrifugation at $12,000 \mathrm{~g}$ for 30 minutes at $4^{\circ} \mathrm{C}$. The lysates were separated by SDS-PAGE and electrophoretically transferred to nitrocellulose membranes (10600002; GE Healthcare, Chicago, IL, USA) in a transbuffer. The membranes were blocked with $5 \%$ non-fat milk in Tris-buffered saline with $0.1 \%$ Tween 20 and incubated with specific primary antibodies, followed by incubation with horseradish peroxidase-conjugated secondary antibodies. The antibodies that were used are given in Table 2. The membranes were visualized with Pierce ECL (32106; Thermo), and Uvitech System (UVITEC,
Cambridge, UK).

\section{Immunofluorescence}

Immunofluorescence was performed as previously described [18]. Isolated enthesis cells were grown on cover glasses and fixed with $10 \%$ formalin for 10 minutes. The cells were then permeabilized with $0.3 \%$ Triton X-100 and in $3 \%$ bovine serum albumin (BSA) in $1 \times$ PBS for an hour at room temperature. The cells were then incubated with the appropriate primary DKK1 antibody in antibody diluent (S3022; DAKO, Carpinteria, CA, USA) overnight at $4^{\circ} \mathrm{C}$. Antibody binding was visualized using the $\mathrm{Cy} 3-$ conjugated anti-rabbit secondary antibody (111-165-144; Jackson Immunoresearch, West Grove, PA, USA). The nuclei were counterstained with DAPI (H-1200; VECTASHIELD, Burlingame, CA, USA). To visualize the stained cells, a fluorescence microscope (Leica Microsystems, Wetzlar, Germany) was used. The primary antibodies that were used are given in Table 2.

\section{Chromatin immunoprecipitation-quantitative PCR (ChIP-qPCR)}

ChIP assays (17-295; Millipore, Burlington, MA, USA) were performed as previously described [19]. In brief, the cells were stimulated in $10 \mathrm{~cm}$ dishes and fixed with a final concentration of $1 \%$ formaldehyde. These cells were sonicated in $1 \times$ PBS with protease inhibitor and then immunoprecipitated with protein A agarose beads conjugated to the phos-NF- $\kappa$ B antibody. The DNA purifica-

Table 1. Primer sequences for $q P C R$

\begin{tabular}{|c|c|c|}
\hline Gene & 5'----Forward-----3' & 5'----Reverse-----'3' \\
\hline GAPDH & GTAACCCGTTGAACCCCATTC & CCATCCAATCGGTAGTAGCG \\
\hline OSTEOCALCIN (OCN) & ATGAGAGCССТСАСАСТССТ & CTTGGACACAAAGGCTGCAC \\
\hline$D K K 1$ & GTGGCCTTCAAGGTGGTAG & АСТСТTGССТСGTССАСТС \\
\hline GAPDH for ChIP & TGCAACACCAACTGCTTAGC & GGCATGGACTGTGGTCATGAG \\
\hline$D K K 1$ for ChIP & GAGCGACTAAGCAAGGGAGG & ACCGCGGCTGCCTTTATAC \\
\hline
\end{tabular}

Table 2. Primary antibodies used in IB, IF, and ChIP

\begin{tabular}{llccc}
\hline \multicolumn{1}{c}{ Antigen } & \multicolumn{1}{c}{ Manufacturer } & Species & Catalog number & Dilution \\
\hline Phos S536 NF- $\kappa$ B & Cell signaling, Denvers, MA, USA & Rabbit & 3033 & $1: 1,000$ (IB) \\
NF- $\kappa$ B & Cell signaling, Denvers, MA, USA & Rabbit & 8242 & $1: 1,000$ (IB) \\
& & & & $1: 50$ (ChIP) \\
DKK1 & Santa Cruz Biotechnology, Santa Cruz, TX, USA & Mouse & Sc-374574 & $1: 100$ (IF) \\
DKK1 & Cell signaling, Denvers,MA, USA & Rabbit & 4687 & $1: 1,000$ (IB) \\
GAPDH & Cell signaling, Denvers, MA, USA & Rabbit & 2118 & $1: 1,000$ (IB) \\
\hline
\end{tabular}

IB: immunoblotting, IF: immunostaining, ChIP: chromatin immunoprecipitation. 
tion was performed using the phenol/chloroform/isoamyl alcohol (P3803; Sigma) method. The DNA was precipitated using $3 \mathrm{M}$ sodium acetate and eluted with DEPC water. Approximately $5 \sim 10$ ng of eluted DNA was used for qPCR. The quantification and calculation of ChIPqPCR have been previously described [20]. The DKK1 promoter sequences were obtained from the Eukaryotic Promoter Database (EPD, http://epd.epfl.ch). The primers used in the ChIP-qPCR are provided in Table 1.

\section{Statistical analysis}

Data are presented as means \pm standard deviations, medians (interquartile range, IQR), or percentages, as appropriate. Differences of age between the two groups were assessed using the Student's t-test. Comparison for serum DKK1 levels between the two groups was assessed by analysis of covariance (ANCOVA) model after adjusting for age. Statistical analyses were conducted using SPSS (version 26.0; IBM Co., Armonk, NY, USA) or GraphPad Prism 6 software. Graphical data representation was performed using GraphPad Prism 6 or 9 software. p-values $<0.05$ were considered statistically significant.

\section{RESULTS}

\section{Serum DKK1 levels were higher in AS than HCs}

AS patients were older than those in the $\mathrm{HC}$ group

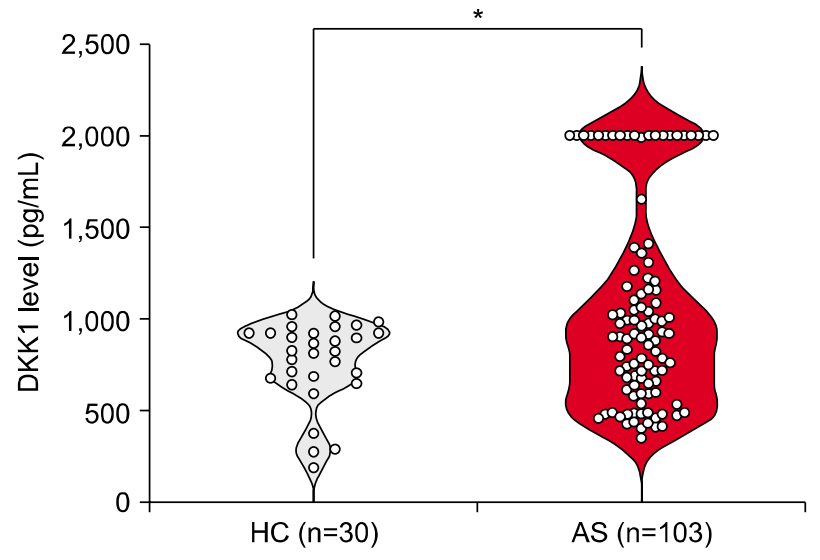

Figure 1. Serum DKK1 levels were higher in ankylosing spondylitis (AS) patents than those of control patients after adjusting for age. The human DKK1 levels were measured in the collected sera (healthy control $[\mathrm{HC}], \mathrm{n}=30 ; \mathrm{AS}, \mathrm{n}=103$ ) using ELISA. Since the classic DKK1 standard curve ranges $0 \sim 2,000$ $\mathrm{pg} / \mathrm{mL}$, we fixed the values as $2,000 \mathrm{pg} / \mathrm{mL}$ if the DKK1 levels go over $2,000 \mathrm{pg} / \mathrm{mL}$. The bars show means \pm standard error of the mean; ${ }^{*} \mathrm{p}<0.05$.
(45.4 \pm 5.0 years vs. $32.1 \pm 5.2$ years, $\mathrm{p}<0.001)$. The median (IQR) DKK1 levels in AS patients were also significantly higher than those of the HCs after adjusting for age (917.4 [615.3 1,310.0] pg/mL vs. 826.2 [670.3 $927.8] \mathrm{pg} / \mathrm{mL}, \mathrm{p}=0.043)$. However, the broad range was shown in the serum DKK1 levels of AS patients (Figure 1).

\section{Treatment of TNF promotes bone formation}

The experimental design and analysis tools for bone formation are shown in Figure 2A. Under bone formation conditions, TNF treatment accelerated matrix maturation (alkaline phosphatase [ALP] and collagen [COL] stains) (Figure 2B) and mineralization (Alizarin Red S rheumatoid arthritis synovium, Hydroxyapatite [HA], and Von Kossa [VON] stains) (Figure 2C and 2D) compared to treatment with vehicle in both control and AS enthesis cells. As shown in Figure 2E, the mRNA and protein expressions of $D K K 1$ and $O C N$ increased after TNF treatment in both control and AS enthesis cells. Notably, the accelerated bone formation and DKK1 expression induced by TNF were pronounced in the AS enthesis compared to those of control.

\section{TNF treatment activates the phosphorylation of $\mathrm{NF}-\mathrm{KB}$ protein to induce $\mathrm{DKK} 1 \mathrm{mRNA}$ expression in enthesis}

Previous studies have suggested that TNF is strongly associated with DKK1 expression in TNF transgenic mice and the rheumatoid arthritic synovium [21,22]. To explore the underlying molecular mechanism of TNF-induced $D K K 1$ expression, we treated control enthesis cells with TNF. After TNF stimulation, the mRNA expression of DKK1 was upregulated compared to those of control (Figure 3A). The TNF-treated enthesis exhibited an increase of the DKK1 protein levels, as revealed by immunofluorescence (Figure 3B). Moreover, the TNF treatment induced the phosphorylated-S536 NF- $\kappa$ B and expression of DKK1 proteins in enthesis (Figure 3C). The activity of wild type (WT) NF- $\kappa$ B promoter was upregulated in a dose-dependent manner of TNF treatment, but not in mutants (S536A and S536E) (Figure 3D). Intriguingly, TNF treatment activated two ( $1 \mathrm{~kb}$ and 0.35 $\mathrm{kb})$ types of the human DKK1 promoter, but showed relatively higher response in the $0.35 \mathrm{~kb}$ DKK1 promoter (Figure $3 \mathrm{E}$ ). Furthermore, the phos-S536 NF- $\kappa$ B protein was enriched at two putative binding sites within the $0.35 \mathrm{~kb}$ DKK1 promoter in response to TNF-treatment (Figure 3F). Conversely, the NF- $\kappa$ B inhibitor (BAY) sup- 
A
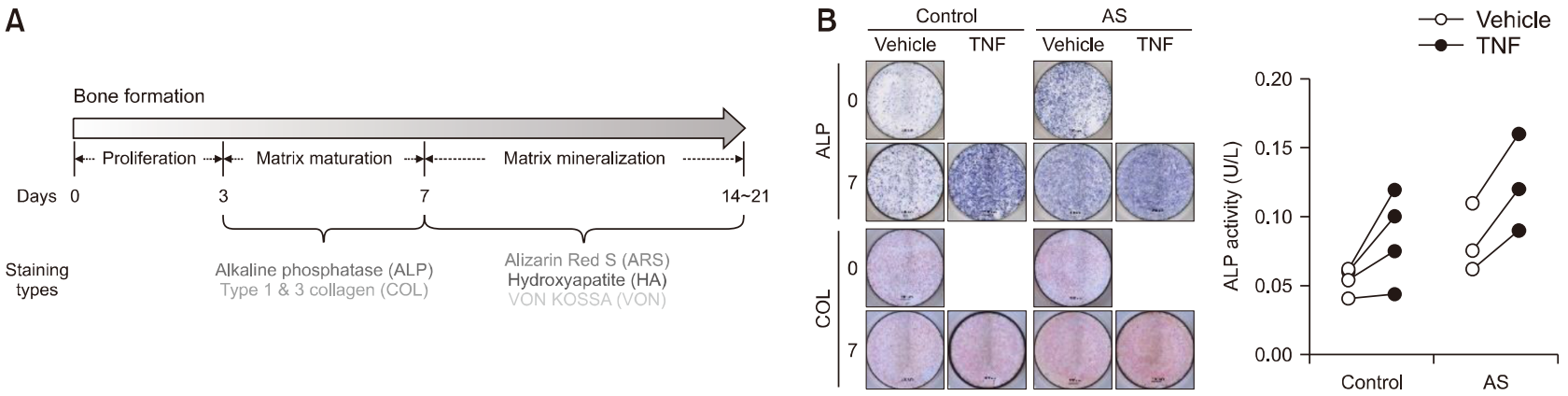

C
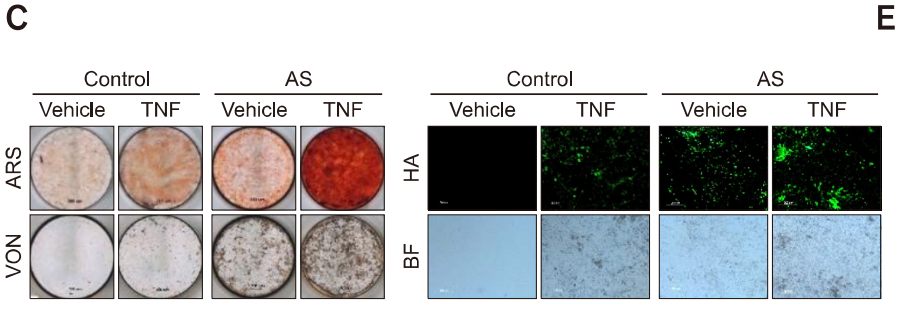

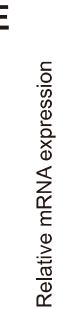
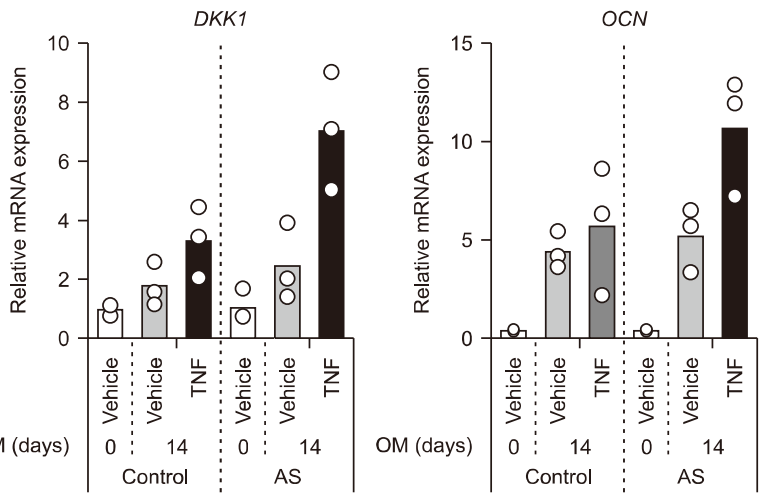

D
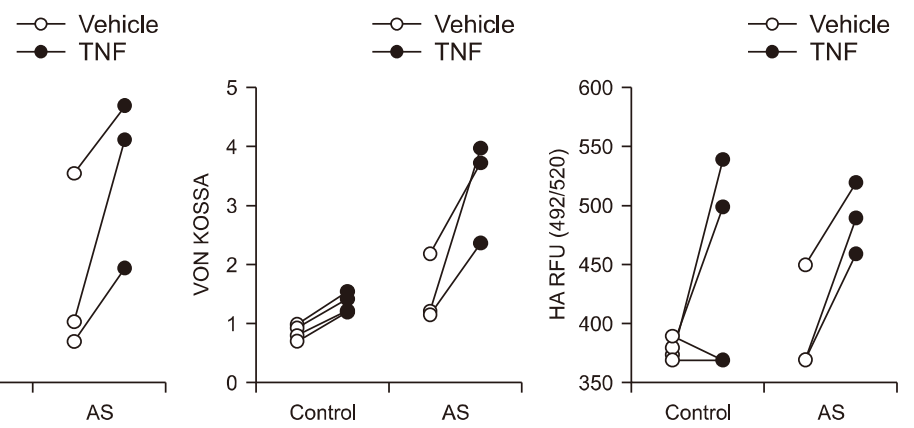

Figure 2. Treatment of TNF promoted bone formation of enthesis. (A) Experimental design for inducing bone formation in primary enthesis cells. Both control- and AS-enthesis cells were followed by bone formation inducing conditional medium for the indicated days. As indicated days, the differentiated cells were subjected to various experimental bone forming activities of (B) ALP and Collagen (COL) staining using Sirius red (left) and intracellular ALP activity (right), (C) Alizarin Red S (ARS), Von Kossa (VON), and Hydroxyapatite (HA) stain images, and (D) Quantification of bone formation indicators stain; ARS, VON, and HA (control=4, $A S=3$ ). All of the staining images is representative of three independent experiments. (E) RT-qPCR and immunoblotting results of differentiated enthesis cells at 14 days (control =3, AS =3). Each dots display individual values. TNF: tumor necrosis factor, AS: ankylosing spondylitis, ALP: alkaline phosphatase.

pressed TNF-induced DKK1 expression by reducing NF$\kappa \mathrm{B}$ phosphorylation (Figure 3G). Taken together, these results suggest that TNF activates NF- $\kappa$ B protein phosphorylation to induce DKK1 mRNA expression in enthesis.

\section{DKK1 overexpression promotes bone formation}

To determine whether DKK1 expression affects bone formation in enthesis, we manipulated DKK1 over- expression in enthesis under bone formation stimulation and observed physiological changes in bone formation. We first confirmed the DKK1 overexpression using RT-qPCR 48 hours after transfection (Figure 4A). There was no significant difference in ALP and COL expression by DKK1 overexpression (Figure 4B), while the calcified nodules and bone formation indicators were increased in DKK1 overexpressed cells compared to controls (Figure 4C and 4D). Taken together, these findings suggest that 
A

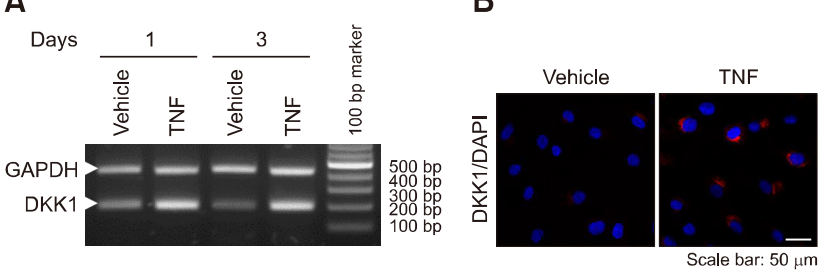

C

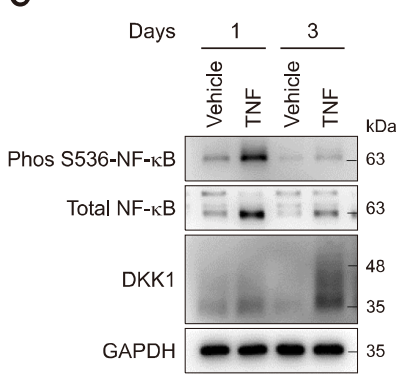

E

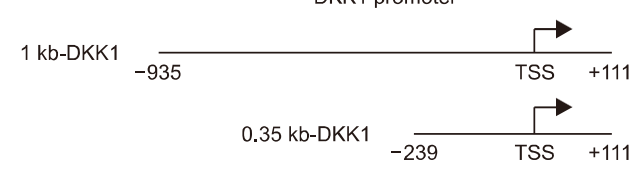

DKK1 promoter

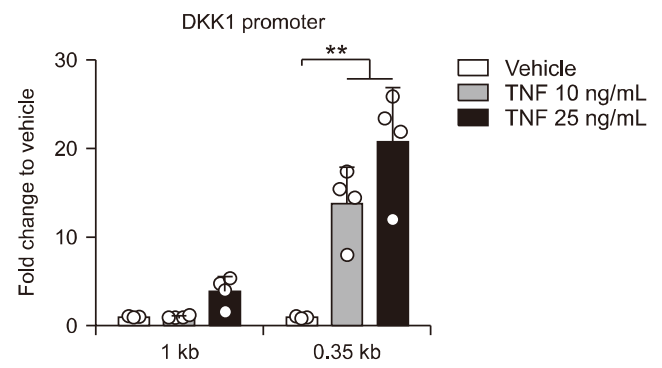

F

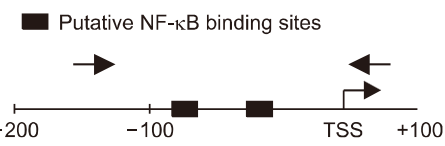

ChIP-qPCR

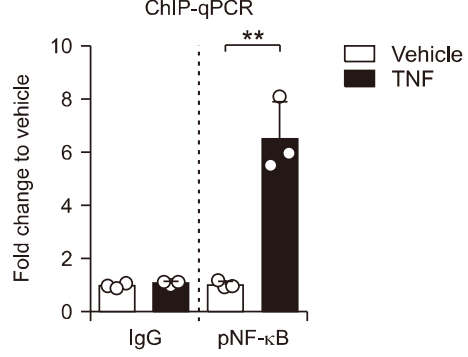

D

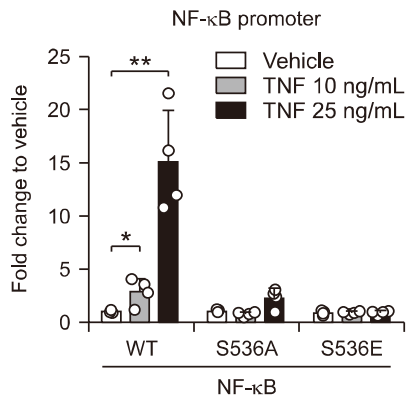

G

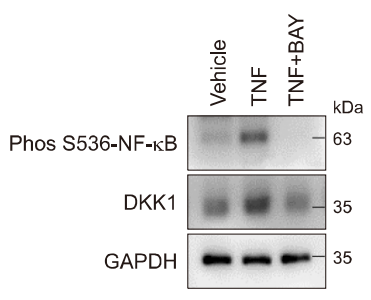

Figure 3. TNF treatment activates NF- $\kappa$ B protein phosphorylation to induce DKK1 mRNA expression in enthesis. Control enthesis cells were stimulated with TNF on days 1 and 3, and analyzed by (A) RT-PCR for DKK1 and GAPDH, and (B) immunofluorescence for DKK1 at day1. (C) Control enthesis cells were stimulated with TNF on days 1 and analyzed by immunoblotting as indicated proteins. (D) Control enthesis cells were co-transfected with three types (WT, S536A mutant, and S536E mutant) of NF- $\kappa$ B, followed by treatment with TNF as indicated doses for 24 hours, and analyzed for luciferase activity $(n=4)$. (E) Control enthesis cells were co-transfected with two types ( $1 \mathrm{~kb}$ and $0.35 \mathrm{~kb}$ ) of the human DKK1 promoter and beta-gal, followed by treatment with TNF as indicated doses for 24 hours, and analyzed for luciferase activity $(n=4)$. (F) Human primary synovial cells were stimulated with $25 \mathrm{ng} / \mathrm{mL}$ TNF for 24 hours, followed by ChIP assays with the phos S536 NF- $\kappa$ B antibody $(\mathrm{n}=3)$. (G) Control enthesis cells were stimulated as indicated for a day and analyzed by immunoblotting. Each dot displays an individual value. Values represent means \pm standard deviations. TNF: tumor necrosis factor, AS: ankylosing spondylitis, ALP: alkaline phosphatase, WT: wild type, ChIP: chromatin immunoprecipitation. ${ }^{*} \mathrm{p}<0.05,{ }^{*} \mathrm{p}<0.01$.

overexpression of DKK1 promotes bone formation in enthesis.

\section{DISCUSSION}

TNF is known to promote bone formation by regulating the expression of various osteogenesis-related genes (such as ALP, RUNX2, and OCN) in various human derived cells such as mesenchymal stem cells, and enthesis cells $[23,24]$, But, it has not been investigated how TNF mediates bone formation in spinal enthesis cells. Here, we reported that AS patients showed higher serum DKK1 levels than those of controls and TNF treatment promoted DKK1 expression and bone formation in enthesis cells. Intriguingly, the TNF-promoted bone formation was more pronounced in AS enthesis cells than those of controls. We also suggested the regulatory molecular mechanism of TNF-induced DKK1 expression in enthesis. DKK1 overexpression in enthesis exhibited an increase in bone formation. Taken together, TNF-induced DKK1 expression is the possible key player in the pathological bone formation of AS.

DKK1 has been recognized as an important regulator of joint remodeling [21]. Due to the bone-forming disease nature of AS, DKK1 has been implicated in the pathophysiology of radiographic AS progression. Interestingly, blocking DKK1 led to sacroiliac joint fusion in an arthritis 

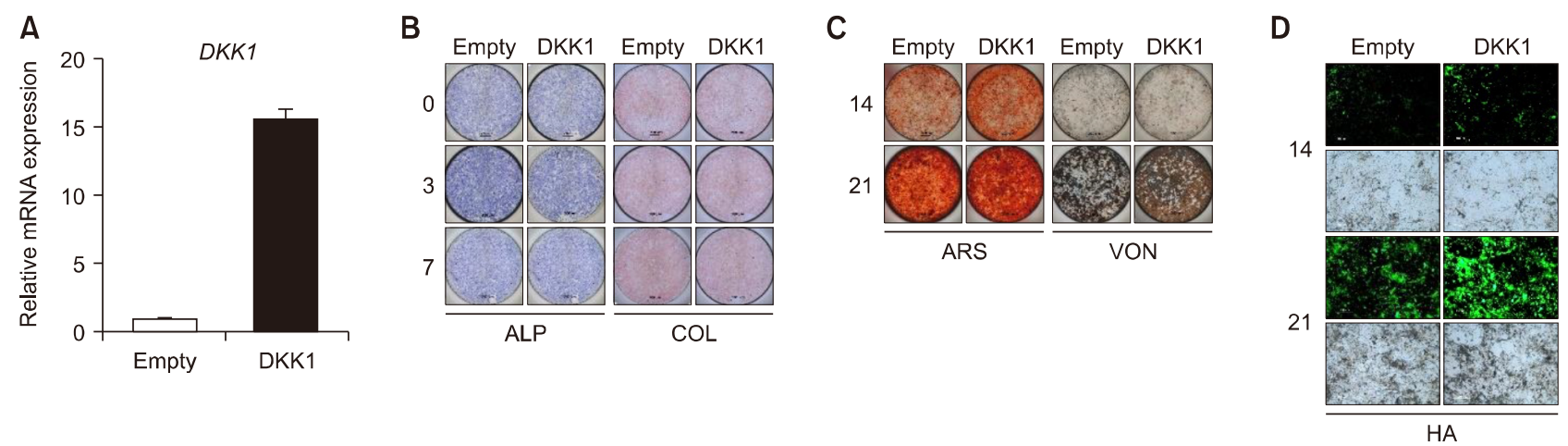

Figure 4. DKK1 overexpression promotes bone formation of enthesis. Enthesis cells were transfected with an empty vector or DKK1 plasmid and then stimulated to osteogenic differentiation. (A) Transfection efficiency was confirmed by RT-qPCR. As indicated days, differentiated cells were subjected to (B) ALP and COL staining, (C) ARS and VON staining, and (D) Hydroxyapatites staining. Representative images are shown. Scale bar is $200 \mu \mathrm{m}$. ALP: alkaline phosphatase, COL: collagen, ARS: Alizarin Red S, HA: hydroxyapatite, VON: Von Kossa.

mice model (TNF transgenic mice), although it did not promote inflammation in the sacroiliac joint [8]. Moreover, a recent study showed that DKK1 significantly reduced the incidence and severity of kyphotic and ankylosing spinal phenotypes in the modified collagen induced arthritis model [25]. Therefore, DKK1 is a well-known potential inhibitor of the Wnt signaling pathway. Elevated DKK1 levels are linked to bone resorption, while decreased levels are associated with new bone formation. However, accumulated data have suggested that DKK1 plays a dual stage-dependent role in osteoblast differentiation. Some studies have shown that DKK1 decreased in extracellular matrix maturation but increased in the mineralization phase of osteoblasts differentiation; therefore, DKK1 is involved in mineralization-related physiological changes [9,26-28]. In this study, we also showed that DKK1 directly promoted bone formation in enthesis. We also identified a discrepancy between our results and the impact of DKK1 on AS-like joint destruction, which was shown in previous studies using murine arthritis models $[8,25]$. This discrepancy may be associated with a difference between experimentation and reality, or between mice and humans. Alternatively, the difference may reflect a dual role of DKK1 or different activities between functional DKK1 and circulating DKK1. Regardless, further studies are needed to close these gaps.

Previous human studies have produced inconsistent results regarding the serum DKK1 levels in AS patients. In some studies, the serum levels of DKK1 were elevated in AS patients compared to controls [29-31], but reduced $[11,32-34]$ or comparable $[10,35,36]$ in other studies.
Thus, relationship between serum DKK1 levels and radiographic changes in AS is still controversial. We found that the serum DKK1 levels of AS were higher than those of controls after adjusting for age. In addition, the DKK1 levels range was wide in AS patients. The previous inconsistence results of the serum DKK1 levels and the wide range of serum DKK1 levels might be associated with a broad range of medical conditions among the patients. Moreover, a recent meta-analysis revealed that the serum DKK1 levels might be associated with the severity of radiological progression or inflammation. In this study, the serum DKK1 levels of AS patients were comparable with that of HCs. However, the serum DKK1 levels in patients with high mSASSS $(>30)$ scores and in patients with abnormal C-reactive protein $(>10 \mathrm{mg} / \mathrm{L})$ were significantly lower than that of HCs. In contrast, the serum DKK1 levels in patients with normal CRP $(\leq 10 \mathrm{mg} / \mathrm{L})$ were increased [13]. Therefore, further studies are needed to investigate the serum DKK1 levels with consideration of the patients' medical conditions.

Few studies have suggested that stimulation of TNF induces DKK1 expression in osteoblasts and synovial cells $[22,37]$, but its underlying mechanism is not elucidated so far. To provide molecular mechanism of TNF-regulating DKK1 expression, $0.35 \mathrm{~kb}$ of human DKK1 promoter activity was significantly increased depending on dose of TNF treatment, suggesting that $0.35 \mathrm{~kb}$ element within DKK1 promoter has a critical role for TNF-induced DKK1 expression (Figure 3E). Additionally, results from ChIP assay showed an increased binding affinity of phos-NF- $\kappa$ $\mathrm{B}$ to two putative sites within $0.35 \mathrm{~kb}$ promoter by TNF treatment (Figure 3F). Taken together, our results sug- 
gest that TNF-activated phos-NF- $\kappa$ B protein binds 0.35 $\mathrm{kb}$ within DKK1 promoter to induce DKK1 transcript level.

The present study has several limitations. First, we compared the serum DKK1 levels between AS patients and HCs. However, we did not consider the medical conditions that may influence the DKK1 levels, such as disease activity. Second, the present study showed that TNF induced DKK1 expression by NF- $\kappa \mathrm{B}$ activation. However, the regulatory molecular mechanisms and the functional role of DKK1 in TNF-promoted bone formation process remain unclear. Despite these limitations, we confirmed that TNF induces DKK1 expression and bone formation using human AS enthesis cells. In addition, we attempted to identify other inflammatory cytokines that regulate DKK1 expression among various inflammatory cytokines related to AS pathophysiology (such as TNF, IL-1B, IL-6, IL-11, IL-17A, IL-23, IL-32, and LPS). Interestingly, only TNF treatment led to DKK1 upregulation in human enthesis cells (data not shown). These data suggest that TNF plays a key role in pathological bone formation, emphasizing anti-TNF treatment can reduce radiographic progression in AS. We also extended the previously reported functional role of DKK1 in bone formation $[26,28]$. We provided novel insights into the molecular linkage between TNF and DKK1. Our results widen fundamental understanding of bone formation and the pathogenesis of radiographic progression in AS.

\section{CONCLUSION}

The serum DKK1 levels of AS patients was higher than that of HCs. The TNF-induced bone formation was remarkable in AS enthesis cells. TNF also induced DKK1 expression by NF- $\kappa \mathrm{B}$ activation. In addition to increased bone formation in DKK1 overexpression, our results suggest that TNF-mediated DKK1 plays a role in the radiographic progression of AS.

\section{ACKNOWLEDGMENTS}

This work was supported by the National Research Foundation of Korea (NRF) grant funded by the Korea government (MSIT) (No. 2019R1A2C2004214 and 2020R1A2C1102386).

\section{CONFLICT OF INTEREST}

No potential conflict of interest relevant to this article was reported.

\section{AUTHOR CONTRIBUTIONS}

S.J., B.N., and T-H.K. designed the study, analyzed the data, and wrote the manuscript. Y.L.L., H.P., and S.W. performed the laboratory work. S-H.C. and Y-S.P. supported the spinal enthesis samples. All authors approved the final version of the manuscript.

\section{REFERENCES}

1. Machado P, Landewé R, Braun J, Hermann KG, Baker D, van der Heijde D. Both structural damage and inflammation of the spine contribute to impairment of spinal mobility in patients with ankylosing spondylitis. Ann Rheum Dis 2010;69:1465-70.

2. Kiltz U, Braun J. Assessments of functioning in patients with axial spondyloarthritis. J Rheum Dis 2020;27:22-9.

3. Schett G, Lories RJ, D'Agostino MA, Elewaut D, Kirkham B, Soriano ER, et al. Enthesitis: from pathophysiology to treatment. Nat Rev Rheumatol 2017;13:731-41.

4. Gratacós J, Collado A, Filella X, Sanmartí R, Cañete J, Llena J, et al. Serum cytokines (IL-6, TNF-alpha, IL-1 beta and IFN-gamma) in ankylosing spondylitis: a close correlation between serum IL-6 and disease activity and severity. $\mathrm{Br} \mathrm{J}$ Rheumatol 1994;33:927-31.

5. Braun J, Bollow M, Neure L, Seipelt E, Seyrekbasan F, Herbst $\mathrm{H}$, et al. Use of immunohistologic and in situ hybridization techniques in the examination of sacroiliac joint biopsy specimens from patients with ankylosing spondylitis. Arthritis Rheum 1995;38:499-505.

6. Koo BS, Oh JS, Park SY, Shin JH, Ahn GY, Lee S, et al. Tumour necrosis factor inhibitors slow radiographic progression in patients with ankylosing spondylitis: 18-year real-world evidence. Ann Rheum Dis 2020;79:1327-32.

7. Maeda K, Kobayashi Y, Koide M, Uehara S, Okamoto M, Ishihara $\mathrm{A}$, et al. The regulation of bone metabolism and disorders by Wnt signaling. Int J Mol Sci 2019;20:5525.

8. Uderhardt S, Diarra D, Katzenbeisser J, David JP, Zwerina J, Richards W, et al. Blockade of Dickkopf (DKK)-1 induces fusion of sacroiliac joints. Ann Rheum Dis 2010;69:592-7.

9. Liu Q, Hu CH, Zhou CH, Cui XX, Yang K, Deng C, et al. DKK1 rescues osteogenic differentiation of mesenchymal stem cells isolated from periodontal ligaments of patients with diabetes mellitus induced periodontitis. Sci Rep 2015;5:13142.

10. Ustun N, Tok F, Kalyoncu U, Motor S, Yuksel R, Yagiz AE, et al. Sclerostin and Dkk-1 in patients with ankylosing spondylitis. Acta Reumatol Port 2014;39:146-51.

11. Liao HT, Lin YF, Tsai CY, Chou TC. Bone morphogenetic proteins and Dickkopf-1 in ankylosing spondylitis. Scand J Rheumatol 2018;47:56-61.

12. Zhang L, Ouyang H, Xie Z, Liang ZH, Wu XW. Serum 
DKK-1 level in the development of ankylosing spondylitis and rheumatic arthritis: a meta-analysis. Exp Mol Med 2016;48:e228.

13. Wu M, Chen M, Ma Y, Yang J, Han R, Yuan Y, et al. Dickkopf-1 in ankylosing spondylitis: review and metaanalysis. Clin Chim Acta 2018;481:177-83.

14. van der Linden S, Valkenburg HA, Cats A. Evaluation of diagnostic criteria for ankylosing spondylitis. A proposal for modification of the New York criteria. Arthritis Rheum 1984;27:361-8

15. Jo S, Won EJ, Kim MJ, Lee YJ, Jin SH, Park PR, et al. STAT3 phosphorylation inhibition for treating inflammation and new bone formation in ankylosing spondylitis. Rheumatology (Oxford) 2020 Nov 25 [Epub]. DOI:10.1093/rheumatology/keaa846.

16. Kim HY, Park JH, Won HY, Lee JY, Kong G. CBX7 inhibits breast tumorigenicity through DKK-1-mediated suppression of the Wnt/ $\beta$-catenin pathway. FASEB J 2015; 29:300-13.

17. Kim H, Chung H, Kim HJ, Lee JY, Oh MY, Kim Y, et al. Id-1 regulates Bcl-2 and Bax expression through p53 and NF-kappaB in MCF-7 breast cancer cells. Breast Cancer Res Treat 2008;112:287-96.

18. Jo S, Wang SE, Lee YL, Kang S, Lee B, Han J, et al. IL-17A induces osteoblast differentiation by activating JAK2/STAT3 in ankylosing spondylitis. Arthritis Res Ther 2018;20:115.

19. Lacazette E. A laboratory practical illustrating the use of the ChIP-qPCR method in a robust model: estrogen receptor alpha immunoprecipitation using Mcf-7 culture cells. Biochem Mol Biol Educ 2017;45:152-60.

20. Wang SE, Ko SY, Jo S, Choi M, Lee SH, Jo HR, et al. TRPV1 regulates stress responses through HDAC2. Cell Rep 2017;19:401-12.

21. Diarra D, Stolina M, Polzer K, Zwerina J, Ominsky MS, Dwyer D, et al. Dickkopf-1 is a master regulator of joint remodeling. Nat Med 2007;13:156-63.

22. Choe JY, Kim JH, Park KY, Choi CH, Kim SK. Activation of dickkopf-1 and focal adhesion kinase pathway by tumour necrosis factor $\alpha$ induces enhanced migration of fibroblast-like synoviocytes in rheumatoid arthritis. Rheumatology (Oxford) 2016;55:928-38.

23. Lavocat F, Osta B, Miossec P. Increased sensitivity of rheumatoid synoviocytes to Schnurri-3 expression in TNF- $\alpha$ and IL-17A induced osteoblastic differentiation. Bone 2016;87:89-96.

24. Osta B, Lavocat F, Eljaafari A, Miossec P. Effects of interleukin-17A on osteogenic differentiation of isolated human mesenchymal stem cells. Front Immunol 2014;5:425.

25. Li X, Wang J, Zhan Z, Li S, Zheng Z, Wang T, et al. Inflammation intensity-dependent expression of osteoinductive Wnt proteins is critical for ectopic new bone formation in ankylosing spondylitis. Arthritis Rheumatol
2018;70:1056-70.

26. Jo S, Yoon S, Lee SY, Kim SY, Park H, Han J, et al. DKK1 induced by $1,25 \mathrm{D} 3$ is required for the mineralization of osteoblasts. Cells 2020;9:236.

27. Li X, Liu P, Liu W, Maye P, Zhang J, Zhang Y, et al. Dkk2 has a role in terminal osteoblast differentiation and mineralized matrix formation. Nat Genet 2005;37:945-52.

28. Nam B, Park H, Lee YL, Oh Y, Park J, Kim SY, et al. TGF $\beta 1$ suppressed matrix mineralization of osteoblasts differentiation by regulating SMURF1-C/EBP $\beta$-DKK1 axis. Int J Mol Sci 2020;21:9771.

29. Tuylu T, Sari I, Solmaz D, Kozaci DL, Akar S, Gunay N, et al. Fetuin-A is related to syndesmophytes in patients with ankylosing spondylitis: a case control study. Clinics (Sao Paulo) 2014;69:688-93.

30. Huang J, Song G, Yin Z, Fu Z, Ye Z. Alteration of bone turnover markers in canonical wingless pathway in patients with ankylosing spondylitis. Arch Rheumatol 2016;31:221-8.

31. Daoussis D, Liossis SN, Solomou EE, Tsanaktsi A, Bounia $\mathrm{K}$, Karampetsou M, et al. Evidence that Dkk-1 is dysfunctional in ankylosing spondylitis. Arthritis Rheum 2010; 62:150-8.

32. Rossini M, Viapiana O, Idolazzi L, Ghellere F, Fracassi E, Troplini S, et al. Higher level of Dickkopf-1 is associated with low bone mineral density and higher prevalence of vertebral fractures in patients with ankylosing spondylitis. Calcif Tissue Int 2016;98:438-45.

33. Kwon SR, Lim MJ, Suh CH, Park SG, Hong YS, Yoon BY, et al. Dickkopf-1 level is lower in patients with ankylosing spondylitis than in healthy people and is not influenced by anti-tumor necrosis factor therapy. Rheumatol Int 2012; 32:2523-7.

34. Korkosz M, Gąsowski J, Leszczyński P, Pawlak-Buś K, Jeka S, Kucharska E, et al. High disease activity in ankylosing spondylitis is associated with increased serum sclerostin level and decreased wingless protein-3a signaling but is not linked with greater structural damage. BMC Musculoskelet Disord 2013;14:99.

35. Sakellariou GT, Iliopoulos A, Konsta M, Kenanidis E, Potoupnis M, Tsiridis E, et al. Serum levels of Dkk-1, sclerostin and VEGF in patients with ankylosing spondylitis and their association with smoking, and clinical, inflammatory and radiographic parameters. Joint Bone Spine 2017;84: 309-15.

36. Niu CC, Lin SS, Yuan LJ, Chen LH, Yang CY, Chung AN, et al. Correlation of blood bone turnover biomarkers and Wnt signaling antagonists with AS, DISH, OPLL, and OYL. BMC Musculoskelet Disord 2017;18:61.

37. Li S, Yin Y, Yao L, Lin Z, Sun S, Zhang J, et al. TNF- $\alpha$ treatment increases DKK1 protein levels in primary osteoblasts via upregulation of DKK1 mRNA levels and downregulation of miR-335-5p. Mol Med Rep 2020;22:1017-25. 